\title{
LOS INVESTIGADORES JUNIOR ESPAÑOLES Y SU IMPLICACIÓN EN LA CIENCIA ABIERTA
}

\author{
Blanca Rodríguez-Bravo* \\ Facultad de Filosofía y Letras, Universidad de León. \\ David Nicholas ** \\ Ciber Research Ltd
}

\begin{abstract}
Resumen: El objetivo es indagar en las actitudes y prácticas de los jóvenes investigadores españoles hacia la ciencia abierta. Se analiza su interés por compartir en abierto publicaciones y datos, por colaborar con otros investigadores y stakeholders, por difundir su investigación y por perseguir el impacto de los resultados científicos.

La metodología se fundamenta en entrevistas y encuestas dirigidas a Early Career Researchers (ECRs) españoles. Los resultados muestran el interés de los investigadores noveles por la ciencia abierta, pero también la necesidad de reconocimiento de las acciones implicadas en ella como requisito para su consolidación entre investigadores en situación precaria. La financiación es también un factor crítico a considerar.

Palabras clave: Ciencia abierta; acceso abierto; datos abiertos; comunicación de la investigación; impacto; jóvenes investigadores; España.

Title: EARLY CAREER RESEARCHERS AND THEIR INVOLVEMENT IN OPEN SCIENCE.

Abstract: The aim is to investigate the attitudes and practices of Spanish Early Career Researchers (ECRs) towards open science. Their interest in sharing openly publications and data, in collaborating with other researchers and stakeholders, in disseminating their research and in looking for results' impact is analyzed.

The methodology is based on interviews and surveys directed to Spanish Early Career Researchers (ECRs).

The results show the interest of novice researchers in open science, but also the need for recognition of the actions involved in it as a requirement for its consolidation among researchers in a precarious situation. Funding is a critical factor to be considered as well.

Keywords: Open science; open access; open data; outreach; impact; early career researchers; Spain.
\end{abstract}

Copyright: (C) 2021 Servicio de Publicaciones de la Universidad de Murcia (Spain). Este es un artículo de acceso abierto distribuido bajo los términos de la licencia Creative Commons Reconocimiento 4.0 Internacional (CC BY 4.0).

\section{INTRODUCCIÓN}

La reputación es el valor principal para un académico (Becher, 1989) y tradicionalmente se ha asociado exclusivamente con la investigación, en concreto con la publicación en revistas revisadas por pares, y con una única medida, como las citas (Jamali, Nicholas, Herman, 2015). Semejante reducida visión limita la consideración de las restantes actividades y productos de los académicos.

En los últimos tiempos, sin embargo, se han producido cambios estructurales en el entorno académico como resultado de la introducción de las tecnologías de la Web 2.0 centradas en facilitar la colaboración y el intercambio de la información. Las nuevas plataformas están modificando la manera de construir, mostrar y medir la reputación en el ecosistema digital. Así lo confirman los resultados de un proyecto encargado por la Comisión Europea para investigar los nuevos desarrollos en el marco de la construcción de la reputación ${ }^{1}$ (Jamali, Nicholas, Herman, 2015; Nicholas et al., 2015; Herman, Nicholas, 2019).

Igualmente, en estos últimos tiempos estamos viviendo la transformación del modelo de ciencia hacia una ciencia abierta. La ciencia abierta supone un cambio de paradigma en el modo de hacer ciencia. Esta no cambia sustancialmente respecto a su motivación y objetivos, pero cambia significativamente respecto a sus métodos. El cambio no reside en lo que se hace sino en cómo se hace. El nuevo modo de hacer ciencia pretende ser abierto, colaborativo y realizado con la sociedad y por ella (Anglada y Abadal, 2018). Estos mismos autores reflexionan en otro trabajo sobre la evolución del concepto (Abadal y Anglada, 2020).

\footnotetext{
*blanca.rodriguez@unileon.es

** dave.nicholas@ciber-research.com
}

Recibido: 26-02-2021; $2^{\mathrm{a}}$ versión: 25-06-2021; aceptado: 06-07-2021.

RODRÍGUEZ BRAVO, B. y NICHOLAS, D. Los investigadores junior españoles y su implicación en la ciencia abierta. Anales de Documentación, 2021, vol. 24, nº 2. Disponible en: http://dx.doi.org/10.6018/analesdoc.470671. 
En otras palabras, los promotores de la ciencia abierta afirman que esta es conocimiento: transparente, accesible, colectivo y cooperativo (Vicente-Saez; Martínez-Fuentes, 2018). Como conocimiento transparente, la ciencia abierta permite que el proceso de investigación sea más eficiente; como conocimiento accesible, la ciencia abierta hace que la investigación esté al alcance de todos; como conocimiento colectivo la ciencia abierta facilita compartir, difundir y participar; y, como conocimiento colaborativo, la ciencia abierta permite la cooperación por medio de herramientas web y el netwworking.

Cuando decimos conocimiento abierto queremos decir libre y reutilizable. Para ser libre se precisa la eliminación de las barreras económicas para acceder a los resultados de la investigación. Para ser reutilizable, la investigación de unos científicos debe poder ser reutilizada por otros.

La investigación científica encuentra muchos obstáculos para poder ser reproducida y verificada debido al hecho de que los artículos científicos no van acompañados de los datos en los que se fundamentan o de la descripción precisa de los procedimientos aplicados para obtener los resultados. La ciencia abierta, facilitando la reproducibilidad de la investigación debería generar una ciencia mejor ya que la accesibilidad y la transparencia traen consigo una mayor eficiencia.

Asimismo, los avances tecnológicos hacen posible la cooperación, y solo la colaboración internacional entre los científicos de todas las disciplinas puede permitirnos afrontar con éxito los grandes desafíos de nuestro siglo. El último de los postulados de base de la ciencia abierta es que la ciencia debe ser hecha con y por la sociedad. La Comisión Europea subraya este aspecto que se descompone en: una ciencia del ciudadano, una ciencia responsable y una investigación científica volcada en responder a los retos de la sociedad (Anglada y Abadal, 2018).

Hasta ahora la ciencia se ha desarrollado en los institutos de investigación con una muy limitada interacción con la sociedad que la financia y a la cual se dirige o debería dirigirse los resultados. La solución utilizada ha sido la divulgación científica, no obstante, la divulgación no basta. En el contexto tecnológico actual es viable instaurar un canal de participación con los ciudadanos y permitir, así, que la sociedad colabore en la construcción de la ciencia.

En este contexto en el que cobra empuje la conexión entre los investigadores y la sociedad buscando el respaldo del ciudadano, ha tenido un papel relevante el Charter \& Code 2 . En enero de 2000, la Comisión de las Comunidades Europeas expresó la necesidad de crear el Espacio Europeo de la investigación como el eje de las acciones futuras de la Comunidad encaminadas a consolidar y estructurar la política de investigación europea. La Carta Europea del Investigador reúne una serie de principios y exigencias generales que especifican el papel, las responsabilidades y los derechos de los investigadores y de las entidades que emplean y/o financian investigadores. El objetivo de la carta es garantizar que la naturaleza de la relación entre los investigadores y los financiadores o empleadores propicie la generación, transferencia, distribución y difusión de conocimientos y avances tecnológicos, así como el desarrollo profesional de los investigadores. Aquí nos limitaremos a destacar dos principios aplicables a los investigadores y vinculados al contenido de este trabajo:

\section{Difusión y explotación de los resultados}

“Todos los investigadores deben velar por que, de acuerdo con sus cláusulas contractuales, los resultados de su investigación se difundan y aprovechen mediante, por ejemplo, comunicados, transferencias a otros contextos de investigación o, si procede, comercialización. De los investigadores expertos, en particular, se espera que tomen la iniciativa para velar porque la investigación sea fructífera y porque los resultados se aprovechen comercialmente y/o se hagan públicos siempre que surja la oportunidad".

\section{Compromiso con la sociedad}

"Los investigadores deben velar por que sus actividades de investigación se den a conocer a la sociedad en general de forma que puedan ser comprendidas por los no especialistas y se mejore así la comprensión de la ciencia por parte del público. El compromiso directo con la sociedad ayudará a que los investigadores comprendan mejor el interés de esta por las prioridades científicas y tecnológicas, así como sus preocupaciones".

Como ya se ha puesto de relieve, la tradicional y convencional forma de establecer y de medir la reputación académica ha ampliado sus límites con los desarrollos de la Ciencia 2.0 que impulsa las distintas maneras de compartir información y de colaborar favorecidas por las redes sociales y las nuevas plataformas. Entre sus consecuencias podemos señalar el hecho de que surge un nuevo tipo de investigador (científico freelance, innovador, ciudadano-investigador, etc.). La investigación, como apuntan Nicholas et al. (2015) ya no es coto cerrado de los académicos. 
Identificar al personal académico como los únicos científicos o investigadores resulta reduccionista y potencia las asimetrías despreciando la contribución de actores extraacadémicos en la investigación. En contextos transdisciplinares las fronteras entre académicos y profesionales se diluyen. Los profesionales o los stakeholders de distintos sectores producen conocimiento $\mathrm{y}$, por tanto, son también científicos e investigadores. Asimismo, los académicos pueden contribuir a dar soluciones prácticas para el mundo real y no se limitan, por tanto, a indagar sobre presupuestos teóricos.

Integrar diferentes métodos y expertos de diversos sectores resulta crucial para resolver problemas complejos (Schmidt, Pröpper, 2017; Luthe, 2017; Fritz, Binder, 2018). En la categorización de las actividades de los académicos que realizan Nicholas y colaboradores y que es resultado del mencionado proyecto financiado por la Comisión Europea, entre un total de 58 actividades clasificadas en cinco dimensiones -investigación, integración, aplicación, docencia y co-creación- se identifican varias en las que juega un papel crucial la colaboración con los profesionales para resolver problemas prácticos con incidencia en la vida del ciudadano (Jamali, Nicholas, Herman, 2015; Nicholas et al., 2015; Herman, Nicholas, 2019).

Los investigadores de ciencias sociales son especialmente proclives a colaborar con los profesionales de su sector en la creación de conocimiento. Esta circunstancia ha sido confirmada en trabajos que ponen de relieve que los científicos de ciencias sociales dan importancia a la difusión de resultados vía redes sociales y blogs, así como a realizar investigación aplicada y consultoría para la industria o la administración (Jamali, Nicholas, Herman, 2015).

\section{OBJETIVOS Y METODOLOGÍA}

Este trabajo busca ahondar en las opiniones y prácticas de la nueva ola de investigadores o Early Career Researchers (ECRs). Trataremos de mostrar en él el peso creciente que otorgan a la apertura y a la transparencia en sus prácticas, a la colaboración con todos los posibles agentes, a la difusión de resultados y a la comunicación al ciudadano. Con este objetivo general, este artículo se propone presentar algunos resultados de un proyecto de índole internacional.

El proyecto Harbingers ${ }^{3}$ se inició en 2016 y persigue determinar si la nueva ola de investigadores está contribuyendo a cambiar el escenario de la comunicación académica. Se pretende conocer si su comportamiento se diferencia del de sus mentores o, por el contrario, continúan los patrones tradicionales. A efectos de este proyecto se entiende por ECRs aquellos investigadores que están realizando el doctorado o bien son doctores pero que no han alcanzado una posición de estabilidad en la academia.

En una primera fase, el estudio longitudinal consistió en la realización de un centenar de entrevistas que se repitieron durante tres años (2016-2018) a investigadores en los inicios de su carrera donde dos terceras partes procedían de ámbitos científicos y una tercera parte de ciencias sociales. Los Early Career Researchers procedían de 7 países: China, España, Estados Unidos, Francia, Malasia, Polonia y Reino Unido.

En este trabajo se hará, asimismo, referencia a un nuevo proyecto, Harbingers- $2^{4}$, iniciado en noviembre de 2020 y que persigue conocer la repercusión de la pandemia en los hábitos y prácticas de comunicación de los investigadores noveles. En el marco de Harbingers- 2 se están realizando también entrevistas a ECRs en los 8 países participantes, los mismos mencionados arriba más Rusia que se incorporó al proyecto Harbingers en 2019. Estas entrevistas se han iniciado en enero de 2021 .

Los datos recabados de las entrevistas de la primera fase de Harbingers (2016-2018) y el análisis realizado de ellos resultaron esenciales para el diseño del cuestionario ${ }^{5}$ elaborado para la segunda fase de Harbingers. Así, el cuestionario se desarrolló con base en el guion de las entrevistas y la experiencia obtenida durante la investigación previa fue esencial para acometer el estudio. Se sabía ya qué preguntar y cómo hacerlo. Para que el cuestionario no resultase tedioso no se recogieron en él todas las cuestiones formuladas durante las entrevistas si no aquellas consideradas estratégicas o que requerían confirmación. El propósito de esta segunda fase era extender el estudio a una población mayor y más diversa de ECRs que incluyera a los científicos de humanidades.

El cuestionario se diseñó en inglés, pero se tradujo a la mayoría de las lenguas nacionales de los investigadores participantes en Harbingers -chino, español, francés, polaco y ruso- para ampliar su radio de alcance y potenciar la respuesta. Se testaron todas las versiones del cuestionario antes de hospedarlo en SurveyMonkey y se abrió el 10 de mayo de 2019 cerrándose la recogida de respuestas el 30 de julio de 2019. 
El cuestionario se iniciaba con una cuestión binaria (sí/no) preguntando a quienes accedían si eran o no ECRs. Si contestaban negativamente su aportación se eliminaba para el análisis realizado. En el caso que nos ocupa, el cuestionario distribuido en español, se obtuvieron 158 respuestas que una vez filtradas y eliminados los 21 investigadores que contestaron no ser ECRs se limitaron a 137 respuestas que se procedió a analizar. Hay que considerar que los encuestados eran libres de contribuir a las preguntas que consideraban oportunas y, por tanto, ninguna pregunta obtuvo el total de respuestas posible (Rodríguez-Bravo, Nicholas, 2020a, 2020b). Las preguntas del cuestionario vinculadas a este estudio se presentan en el Anexo.

\section{RESULTADOS Y DISCUSIÓN}

Los resultados más globales de los datos obtenidos tanto de las entrevistas como de la encuesta se han publicado en diversos trabajos entre los que cabría señalar (Nicholas et al., 2017, 2019a, 2020a, 2020b). En este artículo se presentan resultados relacionados con el interés que en los investigadores noveles españoles han despertado las prácticas de una ciencia abierta y transparente y la colaboración y difusión de su trabajo a todos los stakeholders y a la sociedad.

\subsection{El progresivo calado de la ciencia abierta}

Con frecuencia hemos tenido la sensación de que las decisiones sobre la comunicación científica son tomadas en nombre de los investigadores por políticos, financiadores o editores y se espera que los investigadores las acaten. Parece que algo así está sucediendo en el caso de la ciencia abierta. Es por esta razón que resulta crucial examinar esta, a través de las opiniones de los investigadores noveles que serán quienes habiten ese nuevo universo abierto de la comunicación científica que ahora se está construyendo (Nicholas et al., 2019b).

El proyecto Harbingers, desarrollado entre 2016 y 2019, ha permitido mantener conversaciones anuales sobre la ciencia abierta y sus componentes, principalmente open access, open data y open peer review. Las percepciones sobre actitudes y prácticas obtenidas en las entrevistas (2016-2018) se trataron de contrastar nuevamente con el cuestionario utilizado en 2019 y, actualmente, se está volviendo a indagar sobre estos aspectos en las recién iniciadas entrevistas llevadas a cabo en el marco de Harbingers-2.

El primer resultado que podemos señalar de las primeras entrevistas realizadas es que al preguntar a los ECRs por su opinión acerca de la ciencia abierta estos jóvenes científicos desconocían el concepto. Se recurrió a la definición dada por la Comisión Europea ${ }^{6}$ como principal institución que promueve la ciencia abierta: "Open Science describes the ongoing transitions in the way research is performed, researchers collaborate, knowledge is shared, and science is organised. It represents a new approach to the scientific process based on cooperative work and new ways of knowledge distribution using digital technologies and new collaborative tools".

Ante esta explicación el desconcierto persistía. De ahí que se utilizara la más concreta y descriptiva definición de Eurodoc, European Council for Doctoral Candidates and Junior Researchers: "Open Science is essentially an umbrella term for various practices such as Open Access, Open Data, Open Peer Review, Open Education, Alternative Metrics and Citizen Science". Como veremos a continuación, el número de componentes de la ciencia abierta no se encuentra todavía claramente establecido.

El proyecto Open Science Monitor (Comisión Europea, 2017) utiliza la metáfora de los ejes que hacen girar la rueda de la ciencia abierta y que divide la rueda en tres elementos de base: acceso abierto, datos abiertos y comunicación científica abierta. Por su parte, el proyecto Foster (2018) describe los componentes de la ciencia abierta como las celdas de los panales de las abejas e incluye ocho elementos: open notebooks, datos abiertos, open peer review, open access, software gratuito (open source), redes sociales académicas, ciencia ciudadana y recursos educativos abiertos.

Como hemos apuntado, no todas estas prácticas son consideradas generalmente protagonistas de la ciencia abierta. La más reconocida es la publicación en abierto que con frecuencia se superpone al concepto de la ciencia abierta y se confunde con ella, y también se reconocen en este concepto los datos abiertos y con menos relevancia el open peer review. Acceso abierto y datos abiertos son los dos aspectos que aquí abordaremos en más detalle.

La ciencia abierta debiera ser bien acogida por los ECRs ya que, como han demostrado Herman y Nicholas (2019), ofrece numerosos beneficios potenciales a los investigadores en su posición: a) una superior y más amplia audiencia ya que los trabajos no quedan encerrados en las publicaciones de pago; más lecturas, menciones y recomendaciones en las redes sociales, y, probablemente, más citas de una mayor audiencia; c) incrementa las oportunidades de colaboración; d) se acomoda a las preferencias de los millenials por la transferencia, el compartir y la búsqueda de la igualdad en las reglas del juego; e) proporciona más oportunidades de publicar al incrementar el abanico de publicaciones y algunas 
con una menor exigencia de admisión; f) ofrece mayor facilidad para incrementar su visibilidad, que se halla conectada a la reputación, y es muy tenida en cuenta por las nuevas generaciones.

Sin embargo, pese a apreciar todas las posibilidades mencionadas, los ECRs no tienen tiempo para practicar la ciencia abierta como quisieran. Las entrevistas en el marco de Harbingers permitieron observar que los investigadores noveles en general, y los españoles en particular, tenían poco conocimiento sobre la ciencia abierta. Consecuentemente, tampoco son conscientes de practicarla. Ciertamente, esta situación ha ido evolucionando desde 2016 a 2021, como iremos viendo a continuación. Con todo, la confusión sobre la ciencia abierta, su concepto y sus prácticas persiste. El conocimiento y la aceptación de las revistas en abierto sí es muy superior al conocimiento sobre la apertura de datos o de la alternativa que suponen los informes de arbitraje abiertos al doble ciego como se ha constatado en el trabajo de Rodríguez-Bravo y Nicholas (2020b). Lo que no parece haberse modificado es el poco aprecio e interés mostrado hacia los repositorios, sean institucionales, sean temáticos o especializados en datos como se ha apuntado en Rodríguez-Bravo y Nicholas (2019, 2020a).

Las prácticas de la ciencia abierta se ven muy condicionadas por cuestiones organizacionales, características personales y aspectos sociales. A estos condicionantes se pueden sumar otros como los infraestructurales (Abdullahi, 2019). Asimismo, se ha puesto de relieve que anticipar la recompensa es un factor esencial para promover el conocimiento compartido (Park, Gabbard, 2018). Todo ello sin obviar la influencia de un espíritu altruista como recogen otros estudios (Zenk-Möltgen, Akdeniz, Katsanidou, Nabhoven, Balaban, 2018).

Levin, Leonelli, Weckowska, Castle y Dupré (2016) apuntan que la decisión sobre qué dejar en abierto, cómo y cuándo, varía porque los métodos de investigación, los procesos y los objetivos están muy determinados por el contexto. De ahí que las políticas de open science tengan que ser sensibles a la diversidad de contextos de la investigación a los que tengan que aplicarse. Argumentan que la apertura puede no ser útil y no debe aplicarse indiscriminadamente a todos los estadios de la investigación en todos los campos. Así, Levin y Leonelli (2017) proponen considerar la ciencia abierta como una forma dinámica de interpretar el proceso de investigación y sus resultados que se acompaña de consideraciones económicas además de científicas, culturales, políticas, éticas y sociales.

El reciente informe de la Comisión Europea (2020) referido al progreso de la ciencia abierta señala las barreras que dificultan la adopción de la ciencia abierta: se destaca la necesidad de directrices claras, de recompensa por las prácticas abiertas o la necesidad de formación para acometerlas. Asimismo, se pone de relieve la conveniencia de facilitar y guiar en el proceso de implicar a la ciencia ciudadana en los proyectos de investigación, cuando sea posible. Reconocimiento y financiación son las claves para completar el cambio según Chataway, Parks y Smith (2017), ambos requisitos constatados en este trabajo.

Detallaremos a continuación aquellos ejes de la ciencia abierta más asentados -el open access- o promovidos con más ímpetu -los datos abiertos-.

\subsubsection{Publicar en abierto}

De todas las prácticas de la ciencia abierta es el Open Access (OA) la más conocida por los ECRs, de hecho, a menudo es vista no como un simple componente de la ciencia abierta sino como otro nombre de esta (Nicholas et al., 2019b).

Severin, Egger, Eve y Hürlimann (2018) ponen de manifiesto que el sistema de comunicación académica ha experimentado una transformación desde el acceso cerrado a la cada vez mayor aceptación del acceso abierto que ha superado desde el 2010 el 50\% del total de publicaciones. Constatan también la mayor aceptación de la vía dorada frente a la verde. Otro trabajo que analiza el estado del OA es el de Piwowar, Priem, Larivière et al. (2018). Estos autores que cuantifican la evolución del acceso abierto hasta 2015 dejan constancia del paulatino incremento de las publicaciones en acceso abierto. Curiosamente el mecanismo más utilizado para publicar en abierto no es ni la ruta dorada ni la verde, ni tampoco las revistas híbridas, sino los artículos que se dejan abiertos en la web de los editores sin una licencia open explícita. Esta circunstancia es observada, igualmente, en otros trabajos como el de O'Hanlon, McSweeney y Stabler (2020) quienes han encontrado que parte de los ECRs entrevistados del ámbito de la medicina equiparan el acceso público con el acceso abierto y parecen entender mejor los beneficios del acceso libre a los contenidos que los del acceso abierto.

Severin, Egger, Eve y Hürlimann (2018) confirman que las prácticas de publicación OA varían dependiendo de las disciplinas y tanto en el grado de publicación en acceso abierto como en el canal utilizado para llevarlo a efecto, destacando el compromiso de las ciencias médicas frente al menor predicamento del acceso abierto en las humanidades. Asimismo, en medicina la vía dorada es la predominante, mientras que en humanidades el principal canal para disponer 
contenidos en abierto son las revistas híbridas. Los datos de la encuesta dirigida a los ECRs en 2019 confirman que son los jóvenes científicos de ciencias de la salud los que más difunden sus resultados en acceso abierto (Nicholas et al., 2020c). El estudio reciente de Xu et al. (2020) basado en una encuesta a investigadores chinos señala también diferencias entre disciplinas. Los investigadores de ciencias sociales y humanidades utilizan menos las revistas de acceso abierto que los investigadores de otras disciplinas, aunque están igualmente informados y tienen una actitud hacia ellas igualmente positiva.

Las revistas OA parecían no ser una prioridad para los ECRs en 2016 cuando se comenzó a entrevistarles (Nicholas et al., 2017b) y como confirman Borrego y Anglada (2016) en relación a otros grupos de investigadores. Sin embargo, un trabajo más reciente de Ruiz y Delgado (2018) apunta que esta percepción es heredada de los primeros tiempos del acceso abierto cuando estas revistas carecían del respaldo de las grandes editoriales. Estos autores ponen de relieve la situación ambigua de los investigadores españoles. Aunque desconfían de estas revistas, casi el $70 \%$ han publicado al menos un artículo en los últimos cinco años. La opinión sobre el acceso abierto de los investigadores ha ido mejorando como confirma también el estudio de Segado, Martín y Prieto (2018).

Desde 2016 a 2019 se ha observado también en los investigadores noveles españoles (Rodríguez-Bravo, 2019 y 2020a) un salto cualitativo en interés y en práctica respecto al acceso abierto que obedece a la consideración de los beneficios que una mayor difusión de su investigación traería aparejada. Por su parte, las respuestas del cuestionario de 2019 referentes a las prácticas de publicación ponen de relieve el interés de los ECRs por publicar en revistas bien posicionadas y para establecer ese posicionamiento se guían por el factor de impacto. Este resultado es común al obtenido en otros trabajos sobre el colectivo de jóvenes investigadores (Nicholas et al., 2017b; Rodríguez-Bravo, 2019). Asimismo, este dato se halla en consonancia con el obtenido en el informe Ithaka de US, en el que el $90 \%$ de los científicos americanos que responden afirman compartir sus resultados a través de revistas revisadas por pares, mientras que poco más del 20\% lo hacen en blogs o redes sociales (Blankstein, Wolff-Eisenberg, 2019). Como señala también dicho informe, los investigadores se interesan cada vez más por un modelo de publicación abierto, aunque los incentivos académicos tradicionales continúan motivando su toma de decisiones. De hecho, solo 4 de cada 10 investigadores indican que las ventajas percibidas en las revistas OA sean determinantes a la hora de decidir donde publicar.

Antes de desgranar los resultados específicos del cuestionario distribuido en español conviene hacer referencia al estudio que analiza los datos totales obtenidos de este cuestionario distribuido a nivel global a los jóvenes investigadores y que pueden seguirse en el trabajo de (Nicholas et al., 2020c). Los resultados muestran que la mayoría de los ECRs han publicado en revistas OA pero que la utilización de estas varía en función de la disciplina y del país. Los investigadores aprecian en la publicación en acceso abierto más ventajas que inconvenientes. Entre los últimos, solo el coste es mayoritariamente confirmado, situación constatada en otros estudios como el de Dalton, Tenopir y Björk (2020) o el de O’Hanlon, McSeeney y Stabler (2020).

En el cuestionario distribuido en 2019 (ver Anexo) y dirigido a los investigadores millenial españoles se les pregunta por si publican artículos en acceso abierto. Fueron 100 los ECRs que respondieron a la pregunta correspondiente donde el 44,76\% responde que "sí, frecuentemente", un 37,14\% responde que sí, ocasionalmente" y un 18,10\% contesta negativamente. Las respuestas permiten constatar que una amplia mayoría de los investigadores españoles publican artículos en acceso abierto ya sea con frecuencia, ya sea ocasionalmente (81,90\%) (Rodríguez-Bravo, Nicholas, 2020a).

Son preguntados también por las ventajas de publicar en abierto que les dicta su experiencia. De nuevo son 100 los investigadores que han ofrecido una respuesta. Estos ven en la publicación en acceso abierto una herramienta que incrementa la audiencia y con ella la visibilidad y el impacto además de potenciar la colaboración. En este sentido, Toelch y Ostwald (2018) ponen de relieve que los artículos publicados en acceso abierto consiguen más citas, especialmente si los datos en que se han basado se publican con ellos. Esta percepción es confirmada también en el trabajo de O'Hanlon, McSeeney y Stabler (2020) y en el de Piwowar, Priem, Larivière et al. (2018) quienes indican que los artículos en acceso abierto reciben una media de $18 \%$ más de citas.

Durante las indagaciones realizadas en el seno de Harbingers los investigadores perciben que las publicaciones OA contribuyen a un más rápido avance de la ciencia al diseminar más ampliamente los resultados y publicarlos en plazos más cortos. El cumplimiento con los mandatos de los financiadores y de las instituciones se convierte en otro factor a considerar. La obligación de difundir en abierto las publicaciones producto de proyectos financiados con fondos públicos en España estarían en su origen.

Del mismo modo, se cuestiona a los participantes por las desventajas de publicar en abierto que les dicta su experiencia. En esta ocasión 96 ECRs responden y se señalan dos obstáculos que impiden un apoyo decidido al acceso abierto: el coste de los APCs (Article Process Charges) exigidos y el temor a publicar en una revista depredadora. Los 
investigadores entrevistados entre 2016 y 2018 en España no mencionaban a las revistas depredadoras, aunque desconfiasen de publicar en abierto (Rodríguez-Bravo, Nicholas, 2019). Podría ser que ese concepto como tal haya ido calando relativamente tarde entre los ECRs españoles. Tanto el coste como la supuesta falta de calidad de las revistas son barreras señaladas en otros trabajos como el de O’Hanlon, McSeeney y Stabler (2020).

Desde el inicio de Harbingers en 2016 los investigadores españoles se han ido convenciendo de las fortalezas de la publicación en abierto. La desconfianza ha ido disminuyendo progresivamente. En 2018 los investigadores eran mucho más proclives a publicar en ellas que en 2016. Muchos de ellos habían publicado en alguna revista OA y el recelo previo había disminuido. De hecho, el mayor inconveniente que señalaban era su coste. Hay que considerar que estamos refiriéndonos a jóvenes investigadores que dependen para publicar en abierto del dinero de sus grupos de investigación y de la voluntad de hacerlo o no de sus mentores y/o investigadores principales. En este sentido, el estudio de O'Hanlon, McSeeney y Stabler (2020) alerta de que cuando los investigadores noveles son primeros autores las posibilidades de que ese paper se publique en acceso abierto se incrementan.

El cuestionario de 2019 confirma que los ECRs ya no se muestran firmes en el rechazo a estas revistas por su menor calidad o prestigio. Tampoco tienen peso otros posibles inconvenientes que se mencionaban durante la fase de entrevistas como que puedan facilitar el plagio o sobreexponerles a las críticas. Así, en 2019 son mayoría quienes han utilizado estas revistas en alguna ocasión.

Lo que sí muestran algunos investigadores es la preocupación porque estas revistas estén provocando que las reglas de juego no sean las mismas para todos los aspirantes a puestos permanentes en la ciencia. Los grupos fuertes pueden publicar en abierto lo que reporta una ventaja a sus integrantes en forma de más citas. Como señala una investigadora: "Es una ventaja que no está basada en la excelencia del trabajo presentado o del trabajo previo realizado sino solo en el dinero". Asimismo, el estudio de O'Hanlon, McSeeney y Stabler (2020) señala que el incremento de los APCs puede ampliar la brecha entre aquellos investigadores que disponen de financiación y los demás.

En enero de 2021 se han realizado las primeras entrevistas a jóvenes investigadores en el marco de Harbingers- 2. Los ECRs entrevistados asumen ya con total naturalidad las revistas OA, las ven como una posibilidad que tratan de utilizar para publicar sus papers siempre que disponen de fondos y si hay revistas de esta naturaleza posicionadas en lo más alto del ranking de los JCR. Si no es así, acudirán a ellas solo para publicar trabajos que no puedan optar a las revistas más reputadas.

Respecto a los repositorios y a los servidores de preprints, estos no recaban interés, y en alguna ocasión obtienen un claro rechazo. Los ECRs españoles no consideran que un producto depositado en estas plataformas deba tener la consideración de una publicación que ha sido sometida a un serio proceso de revisión por pares. Lo consideran un atajo en la carrera científica. Sin embargo, un reciente estudio en el ámbito de la Biología, tomando como referencia el uso de bioRxiv refleja que publicar un preprint puede incrementar la atención y las citas que reciba el posterior artículo revisado (Fu, Hughey, 2019). Tal vez los investigadores noveles españoles cambien de actitud sobre estas herramientas, pero lo cierto es que entre 2016 y 2021 no se han apreciado avances. El escaso entusiasmo por el autoarchivo puede estar en la raíz del moderado crecimiento del acceso abierto que se ha observado en los últimos años (O'Hanlon, McSeeney y Stabler, 2020).

\subsubsection{Compartir datos}

Hablar de datos abiertos en la investigación significa hablar de compartir datos que implica hacer disponibles nuestros datos para que otros los utilicen. Los avances tecnológicos incrementan las oportunidades de compartir datos dado que facilitan su colecta, almacenamiento y análisis.

Los datos compartidos promueven la innovación puesto que estos pueden ser usados con distintos fines: permiten la colaboración entre los creadores de los datos y otros investigadores interesados en su utilización; maximizan la transparencia de los datos y la confianza que se les concede; facilitan la reproducibilidad de los experimentos; permiten que los resultados de la investigación sean verificados; facilitan la reutilización de datos; reducen costes evitando la duplicación de datos; incrementan el impacto y la visibilidad de la investigación; promocionan los proyectos de investigación de los que derivan los datos y las publicaciones; y generan reconocimiento a los investigadores que producen los datos.

La Comisión Europea ha sido el mayor promotor de los datos abiertos de investigación en el contexto de la ciencia abierta. Este organismo subraya ${ }^{7}$ que el valor de los datos de investigación no es solo científico, sino que es considerado clave para el progreso económico y la innovación. Sin embargo, la literatura muestra que compartir datos es una práctica 
poco común entre los académicos que puede obedecer a la falta de información o a que los investigadores tienen todavía dudas sobre la conveniencia de poner a disposición libremente sus datos para que otros investigadores los utilicen.

No cabe duda de que para que exista una práctica es necesario una actitud proclive a ella y para que esto pueda darse lo primero es conocer su existencia. Aparentemente todavía la información sobre datos abiertos y datos compartidos no es común. Los factores que pueden promover estas prácticas serían: los mandatos de las agencias financiadoras, las políticas de los editores de revistas; la política de la institución y las circunstancias particulares de la disciplina de cada investigador que puede ser receptiva a estas prácticas o contraria. Por último, otro factor a considerar es la popularidad de los repositorios de datos.

La influencia de los postulados de los financiadores ha sido recogida en trabajos como el de McGuire, Hamilton, Lunstroth, McCullough y Goldman (2008) quienes confirman que la existencia de mandatos supone una presión en los investigadores que determina sus prácticas referidas a compartir datos. Asimismo, la influencia de la política de las revistas es confirmada en los estudios de Piwowar y Chapman (2010) y de Weber, Piwowar y Vision (2010) quienes aprecian una correlación positiva entre la existencia de una política respecto al data sharing en las revistas y la frecuencia con que los investigadores hacen disponibles sus datos en repositorios.

También son determinantes las acciones emprendidas por las instituciones en relación a promover la apertura de datos como ya señalaba Wouters (2002). Igualmente, varios estudios han mostrado que las prácticas compartiendo datos difieren entre las disciplinas en gran medida (Kim; Nah, 2018). La existencia de directrices institucionales o disciplinares facilitan la activación de la práctica de datos abiertos. Por último, la existencia de repositorios de datos en las instituciones impulsa la práctica de compartir datos como han señalado Cragin, Palmer, Carlson y Witt (2010); y Marcial y Hemminger (2010). La presencia de plataformas que faciliten y optimicen el uso de los datos conecta con otros aspectos como es la formación precisa para organizar y etiquetar datos o la conectividad, factores que condicionan las prácticas de los investigadores (Van den Eynden, Corti, 2017; Cao, Giyyarpuram, Farahbakhsh, Crespi, 2017; Roberts, Anderson, Skerratt, Farrington, 2017).

Las principales motivaciones de los académicos para compartir datos serían, de un lado, el considerar la apertura de los datos como un paso adelante en el entorno académico, contemplándolo como una práctica de apertura y de transparencia de los materiales que puede contribuir al avance de la investigación científica; de otro lado, los académicos consideran que compartir datos es una oportunidad de ayudar a otros académicos en su esfuerzo por construir su propia investigación. Todas las motivaciones, no son, sin embargo, altruistas, los investigadores persiguen también la obtención de más citas, colaboraciones, reputación, promoción, incentivos o la protección de los datos de usos dudosos. Así, aquellos académicos que perciben beneficios en el hecho de compartir los datos están dispuestos a involucrarse más en esta práctica como han recogido diversos estudios previos (Kim, 2017; Nosek et al., 2015).

Las barreras principales que dificultan compartir datos son principalmente las siguientes: los académicos consideran arriesgado compartir los datos si todavía no han publicado los trabajos derivados de ellos. Quieren asegurarse que son los primeros en conseguir los resultados que esos datos han posibilitado y quieren maximizar su uso. Tampoco se sienten cómodos con la posibilidad de mostrar datos erróneos o mal anonimizados (Takashima et al., 2018); consideran, asimismo, que compartir datos implica esfuerzo y tiempo dado que es preciso limpiar, organizar y etiquetar los datos antes de subirlos al repositorio y, frecuentemente, sacrificar más tiempo ofreciendo explicaciones a otros investigadores para que comprendan el contexto de los datos. Ya Tenopir et al. (2011) apuntaban a la escasez de tiempo y de financiación para arreglar los datos antes de subirlos a una plataforma, como una razón que limitaba la apertura de datasets.

Por tanto, a los investigadores les gustaría compartir datos con condiciones que les garantizase la seguridad y la privacidad de estos, los privilegios en la publicación de resultados y el reconocimiento del origen de los datos. Esta realidad es constatada en los resultados recabados recientemente en la encuesta realizada por Digital Science (2020) donde destacan las citas, el impacto y la visibilidad como las principales motivaciones para compartir datos. Respecto a las principales causas de preocupación se encuentran: el mal uso de los datos; la posible existencia de información sensible y que requiera permisos antes de ser compartida; no recibir el crédito y reconocimiento apropiado; la inseguridad sobre las licencias de copyright o la duda sobre si se tiene permiso para compartir los datasets. Con un peso considerable se señalan también otras razones: el coste de compartir datos, el trabajo que supone organizarlos de un modo utilizable, la falta de tiempo para depositarlos; y el hecho de querer seguir explotándolos.

A continuación, se ofrecen en detalle los datos obtenidos de los investigadores noveles españoles. En las entrevistas realizadas entre 2016 y 2018 fue en este último año cuando se introdujo una pregunta sobre la experiencia de los jóvenes 
investigadores compartiendo datos. Se constató que la experiencia era muy limitada y que el convencimiento acerca de practicar la apertura de los datasets no era decidido (Rodríguez-Bravo, Nicholas, 2019).

Respecto a la encuesta de 2019 (Anexo 1), la primera pregunta relativa a datos abiertos se dirigía a conocer si los ECRs habían producido datos. La pregunta fue respondida por 97 investigadores. El 60,82\% respondieron afirmativamente y el 39,18\% lo hicieron negativamente. A quienes contestaron afirmativamente se les preguntaba si los habían dejado disponibles en abierto. Solo el 41,94\% respondieron afirmativamente. Un 58,06\% lo hicieron negativamente. Se constata, por tanto, que la práctica de abrir los datasets propios para otros investigadores no se habría generalizado todavía.

Se indaga a continuación sobre los medios utilizados para hacer disponibles los datos permitiendo señalar varias respuestas. Respondieron un total de 28 investigadores, un número muy escueto que ya dice mucho del desconocimiento que los investigadores tienen sobre esta práctica. La respuesta más apoyada $(67,86 \%)$ era la que señalaba la publicación de los datos como material suplementario de un artículo, seguida por el alojamiento de los datos en un sitio web (42,86\%) o en repositorios de datos como Dryad, Figshare o Zenodo (39,29\%), y la menos frecuente publicación de un data paper independiente $(10,71 \%)$.

Seguidamente, se preguntó por los motivos que les impulsaron a dejar accesibles los datos y se les pidió que eligieran un máximo de tres razones entre nueve respuestas ofrecidas. En esta ocasión solo respondieron 26 investigadores. Permitir la reproducibilidad y la reutilización son las opciones más apoyadas ambas con el 61,54\%. A continuación, reciben el apoyo de la mitad de quienes respondieron la finalidad de su preservación y futura accesibilidad, así como su consideración como una señal de credibilidad dado que permite comprobar que la investigación realizada ha sido cuidadosa. Facilitar la colaboración y el compromiso con las políticas del acceso abierto son también razones de peso para el $46,15 \%$ de quienes han contestado a esta pregunta. Los mandatos de la ciencia abierta y la política de publicación de las revistas son menos determinantes con un 38,46\% y un 30,77\% respectivamente de investigadores que las han señalado. Finalmente, no parece considerarse que suponga una ventaja para la obtención de citas, ya que solo dos investigadores $(7,69 \%)$ han señalado esta respuesta, aspecto que sí es prioritario en la consideración de optar por publicar en acceso abierto. Como ya hemos apuntado, este resultado no confirma el hallado en otros estudios como son los de Kim (2017) o Nosek et al. (2015). Tampoco es acorde con los datos recogidos en la encuesta realizada por Digital Science (2020).

Igualmente, la última pregunta relativa a los datos se dirigía a quienes no los ponían a disposición de otros investigadores. Se les pedía que seleccionaran un máximo de tres opciones de las seis elencadas. Esta pregunta fue respondida por 31 investigadores. La explicación más apoyada es la relativa a que los datos no son elegibles para ser puestos a disposición de otros investigadores, debido a su carácter confidencial o relacionado con la seguridad o motivos similares. El 61,29\% de quienes respondieron se escudaban en esta razón. Menos apoyo recibieron respuestas vinculadas como que el tamaño de los archivos de datos impide compartirlos (16,13\%). Un 35,48\% responden también que si no comparten los datos es porque no existe un mandato que les obligue. Se alega igualmente que limpiar los datos para compartirlos supone demasiado trabajo (22,58\%). Asimismo, se aprecia que se considera arriesgado compartir los datos con otros investigadores. Así un 35,48\% afirma estar preocupado por la posible competencia y un porcentaje idéntico indica un riesgo potencial para el desarrollo de su carrera dado que compartir datos no es recompensado por los sistemas de evaluación. Muchas de estas respuestas sí se relacionan con las obtenidas en el cuestionario mencionado ya de Digital Science (2020).

En una pregunta abierta que los investigadores tenían disponible, seis científicos aportaron otras razones relativas al desconocimiento sobre cómo hacerlo o la falta de oportunidad. Otro investigador considera que cuesta demasiado obtener datos como para compartirlos. Hay quien duda que los datos puedan tener utilidad para otro investigador por ser específicos y difíciles de interpretar. Por último, una respuesta ponía de relieve que con la actual ley de protección de datos podría incurrirse en un delito si se comparten determinados datos.

Ya en 2018 en la fase de entrevistas se apreciaba cierta indecisión en los investigadores entrevistados. En teoría eran favorables a la apertura de datos, pero en la práctica deseaban explotarlos al máximo ellos antes de compartirlos. Este parece ser el sentir todavía como se ha podido constatar en las entrevistas realizadas en enero de 2021. Tampoco parece que los investigadores estén muy al tanto de la práctica en sí misma ni de las herramientas a utilizar. La mayoría no conoce ni ha utilizado repositorios de datos. Quizá la inclusión del requisito de definir un plan de gestión de datos en la convocatoria de proyectos de I+D+i del Ministerio de Ciencia e Innovación en 2020 haga que los investigadores vayan familiarizándose con esta práctica, su alcance y significado. 


\subsection{El impacto de la investigación, una prioridad para los ECRs}

En los años de vigencia de Harbingers y en los que se realizaron las entrevistas a este colectivo de ECRs (20162018) se observó cómo concedían cada vez más importancia al impacto de su labor investigadora (Rodríguez-bravo, Nicholas, 2019). El impacto es una prioridad absoluta en 2018. Este colectivo persigue que su investigación llegue a sus colegas, a las autoridades académicas, a quienes fijan las políticas de investigación, a la industria y a la sociedad en general. Un investigador resume su interés en tener impacto en los siguientes colectivos: "los colegas para obtener su colaboración, las autoridades académicas para obtener ayuda financiera y la sociedad para ganar su respeto y apoyo".

Los investigadores creen en los beneficios que les puede aportar la colaboración con otros investigadores de su área, de otras áreas y de otros países. Básicamente porque pueden aprender de otros, les permite utilizar técnicas más avanzadas, y ello redundará en mejores trabajos y en mayor impacto: "La colaboración mejora el impacto porque permite usar mejores técnicas, aprender más y publicar mejores trabajos en mejores revistas", señala una investigadora. Es el "efecto dominó", como lo denominó otra investigadora en 2016. No obstante, siempre hay quien es menos positivo: "Colaborar extensivamente te puede hacer perder las posiciones principales: primer autor, último o autor de correspondencia”.

Asimismo, los investigadores que trabajan en ciencia aplicada aspiran a que su trabajo pueda ser útil para la industria. La transferencia de conocimiento es esencial y las patentes parece que adquieren más valor en las evaluaciones. Una de las investigadoras actualmente en Reino Unido señala que en este país con REF (Research Excellence Framework) "la agenda de impacto es fundamental”. En la entrevista realizada en enero de 2021 la misma científica detalló la intensa labor que se lleva a cabo en Reino Unido para comunicar el significado de la investigación a la sociedad, las vías utilizadas son numerosas incluyendo dirigirse a los consumidores en las grandes superficies.

Conseguir el impacto necesario requiere de una labor previa de difusión y los investigadores cada vez están más convencidos de la utilidad para incrementar su reconocimiento de mostrar y compartir sus publicaciones a través de las redes sociales y de publicar en abierto. Las redes sociales como ResearchGate, pero también Twitter, han ido cobrando mayor relevancia que en 2016 cuando fueron entrevistados por primera vez. Gestionar correctamente la imagen digital empieza a preocupar a estos investigadores que son más conscientes de las ventajas que pueden obtener si invierten más tiempo en gestionar su identidad en estas plataformas: "Este año me he abierto una cuenta profesional en inglés en Twitter", indica una investigadora, y otro investigador apunta: "Las redes sociales dan visibilidad, pero la mayor exposición de nuestro trabajo puede incrementar la reputación porque puedes obtener citas, invitaciones, nuevos contactos, etc.".

Las redes sociales académicas, no obstante, se siguen utilizando principalmente para compartir publicaciones, pero también para descubrir y acceder a la investigación de los colegas, participar en discusiones, etc. En el cuestionario respondido en 2019 se observa que además de para compartir la investigación y para interactuar con los pares existe una tercera finalidad que es mantenerse actualizados en el campo de investigación. La persecución de esa diferencia en el currículum que les permita prosperar sigue estando muy vinculada a la obtención de citas. Ser citado es equivalente a tener impacto entre los iguales. No obstante, el desconocimiento de los temas de copyright les retrae de compartir sus publicaciones en las redes sociales y/o comunidades online en muchos casos: "El principal escollo para compartir publicaciones reside en las restricciones de copyright de los editores que no facilitan el subir textos completos a las redes sociales".

En términos de reputación, algunos investigadores son conscientes de que no pueden limitarse a obtener proyectos financiados y a publicar pues persiguen integrarse en un proceso de colaboración constructiva que les conduzca a la transformación social. Los investigadores cada vez valoran más que su trabajo redunde en la mejora de la vida del ciudadano y así lo ponen de relieve los ECRs entrevistados tanto los científicos como los investigadores de ciencias sociales.

Los resultados globales de Harbingers que miden los cambios en actitudes y prácticas de los ECRs entrevistados entre 2016 y 2018 ponen de relieve que los principales cambios son los que se han experimentado en los apartados de colaboración y de impacto y tanto en actitudes como en prácticas (Nicholas et al., 2019a). En el caso español, los ECRs sienten, asimismo, ese interés y preocupación creciente por conseguir el respaldo de la sociedad a su trabajo como científicos, así como por colaborar con empresas e instituciones de todos los sectores en la creación y difusión del saber. El verdadero impacto, como apuntan los ECRs españoles, es contribuir a la mejora de la vida del ciudadano y para ello el primer paso es potenciar la comunicación, divulgación y cooperación (Rodríguez-Bravo, 2019). 
En las entrevistas realizadas en 2017 y 2018 se ha apreciado un interés creciente por conseguir que la investigación llegue a la sociedad, por comunicar lo que se está haciendo con los impuestos de los ciudadanos a los ciudadanos. Se pone de relieve que conseguir la mejora de la vida de los mismos ha de ser un objetivo prioritario. Un investigador apunta que "construir puentes con la sociedad es una obligación".

Acercar la ciencia a niños y jóvenes también se resalta como objetivo prioritario. Se mencionan distintas actuaciones en diferentes contextos como es el caso de la Semana de la Ciencia, la Expociencia, la Noche Europea de los investigadores, el Día Mundial de la Mujer y la Niña en la Ciencia, Stem Talent Girl, la actividad Pint Of Science o Tesis en 3 Minutos. También se apuntan visitas de escolares a los centros de investigación como vía para transmitir la pasión por la ciencia a este colectivo todavía muy permeable.

Del mismo modo, se indica la participación en los grupos de ciencia de los partidos políticos como una vía idónea para orientar la política científica y potenciar el valor percibido de los científicos en la sociedad: "Hay que conseguir que los políticos sean conscientes de la importancia de la ciencia y de que obtener resultados lleva tiempo”.

No obstante, esta motivación generalizada por incidir en la transmisión de la ciencia a la sociedad no se plasma en acción tanto como les gustaría, porque no se le da prioridad dado que, como señala un investigador: "aunque la difusión de la investigación es un objetivo de HORIZONTE 2020, todavía no se ha considerado un indicador para la evaluación del curriculum vitae del científico". Las entrevistas realizadas en enero de 2021 apuntan en la misma dirección. No existen suficientes estímulos. Los investigadores noveles consideran que la inclusión de planes de difusión en las convocatorias, por ejemplo, en las de solicitud de proyectos, es algo formal y sin transcendencia en la evaluación final que se realiza.

En 2021 los ECRs tienen mayoritariamente el deseo de difundir su investigación fuera de la academia, pero no disponen del tiempo necesario para dedicarse a ello tanto como desearían ni saben cómo maximizar el esfuerzo invertido. Abogan por que se ofrezca apoyo institucional para comunicar a los diferentes colectivos interesados y que estas acciones sean valoradas en las acreditaciones.

\section{CONCLUSIONES}

Los ECRs españoles, si bien afirman hacer un esfuerzo por adoptar los principios de la ciencia abierta y compartir la investigación no parece que en la práctica lo lleven a efecto de manera muy decidida. Se muestran cautos a la hora de compartir resultados no publicados, se limitan a compartir en redes sociales preprints y no muestran interés por publicar en abierto utilizando los repositorios institucionales o temáticos. En el apartado de publicación en abierto el progreso se ha notado más que en el de la apertura de datos. Con todo, no podemos olvidar que las prácticas de los ECRs en relación con la ciencia abierta no son totalmente suyas, muchas decisiones dependen de sus coautores o de los investigadores principales.

Desde 2018 los investigadores noveles, pese a su focalización en la publicación en revistas de alto factor de impacto, procuran diversificarse para poder responder a cualquier nueva exigencia solicitada para acceder a los nuevos contratos y aunque no practiquen como quisieran están convencidos que el sistema de la comunicación científica del futuro ha de ser más flexible, abierto y transparente. Asimismo, ha de estar más comprometido con la comunicación de resultados a todos los públicos.

Los ECRs españoles se muestran cada vez más positivos en relación con sus posibilidades como agentes de cambio en el modelo de comunicación científica. Y sus sugerencias se refieren a compartir los avances científicos con el ciudadano en un lenguaje comprensible para este, a colaborar con otros investigadores en vez de competir, a difundir su investigación libremente en abierto y no solo a través de revistas de impacto, a que la evaluación del científico no se limite a publicaciones y proyectos. Desearían implicarse mucho más en la difusión de la ciencia a todas las capas de la sociedad, así como en la colaboración con otros agentes que consideran una necesidad en un entorno cada vez más global y transdisciplinar.

Las revistas en acceso abierto están ya integradas en los hábitos de comunicación de los investigadores. Más camino queda por recorrer en la promoción de los repositorios o respecto a la práctica de abrir los datos. La difusión a la sociedad se considera un deber que gustaría realizar si las instituciones y las agencias evaluadoras reconocen estas acciones que implican un tiempo del que no se dispone. 


\section{NOTAS}

1 "Analysis of Emerging Reputation Mechanisms for Scholars", 2014-2015.

${ }^{2}$ Recomendación de la Comisión de 11 de marzo de 2005 relativa a la Carta Europea del Investigador y al Código de Conducta para la Contratación de Investigadores. $<$ https://euraxess.ec.europa.eu/jobs/charter $>$.

$3<$ http://ciber-research.uk/harbingers.html $>$.

$4<$ http://ciber-research.com/harbingers-2/>.

$5<$ http://ciber-research.uk/download/ECR-questionnaire-for-website-20191129.pdf $>$.

${ }^{6}<$ https://ec.europa.eu/research/openscience7index.cfm?pg=open-science-policy-platform-faqs $>$. Comisión Europea. Recomendaciones para el acceso y la preservación de la información científica (25 de abril de 2018).

${ }^{7}$ Comisión Europea. Recomendaciones para el acceso y la preservación de la información científica (25 de abril de 2018$)$.

\section{BIBLIOGRAFÍA}

ABDULLAHI, K.A. Data sharing practices of academics in Nigeria: the effects of organizational culture. (Tesis doctoral). Kuala Lumpur: Faculty of computer Science and Information Technology, University of Malaya, 2019.

ABADAL, E. y ANGLADA, L. Ciencia abierta: cómo han evolucionado la denominación y el concepto. Anales de Documentación, 2020, vol. 23, nº 1. Disponible en: http://dx.doi.org/10.6018/analesdoc.378171.

ANGLADA, L. y ABADAL, E. ¿Qué es la ciencia abierta? Anuario ThinkEPI, 2018, vol. 12, p. 292-298. Disponible en: https://doi.org/10.3145/thinkepi.2018.43.

BECHER, T. Academic Tribes and Territories: Intellectual Enquiry and the Cultures of Disciplines. Bristol. Society for Research into Higher Education and Open University Press, 1989.

BLANKSTEIN, M. y WOLFF-EISENBERG, C. Ithaka S+R US Faculty Survey 2018: Report, April 12, 2019. Ithaka S+R. Disponible en: <https://sr.ithaka.org/wp-content/uploads/2019/03/SR-Report-US-Faculty-Survey-201804122019.pdf> [Consulta: 28 de enero de 2021]

BORREGO, A. y ANGLADA, L. Faculty information behavior in the electronic environment: Attitudes towards searching, publishing and libraries. New Library World, 2016, vol. 117, nº 3/4, p. 173-185.

CAO, Q.H.; GIYYARPURAM, M.; FARAHBAKHSH, R. y CRESPI, N. Policy-based usage control for a trustworthy data sharing platform in smart cities. Future Generation Computer Systems, 2020, vol. 107, p. 998-1010.

CHATAWAY, J.; PARKS, S. y SMITH, E. How Will Open Science Impact on University-Industry Collaboration? Foresight and STI governance, 2017, vol. 11, n 2, p. 44-53.

COMISIÓN EUROPEA. Open science monitor. 2017 Disponible en: $<$ https://ec.europa.eu/research/openscience7index.cfm?pg=home\&section=monitor $>$ [Consulta: 2 de enero de 2021]

COMISIÓN EUROPEA. Recomendación (UE) 2018/790 de la Comisión de 25 de abril de 2018 relativa al acceso a la información científica y a su preservación. Diario Oficial de la Comisión Europea, 2018. Disponible en: $<$ https://eurlex.europa.eu/legal-content/ES/TXT/PDF/?uri=CELEX:32018H0790\&from=ES> [Consulta: 8 de enero de 2021]

COMISIÓN EUROPEA. Progress on Open Science: towards a shared research knowledge system: final report of the Open Science Policy Platform. Luxembourg: Publications Office of the European Union, 2020.

CRAGIN, M.H.; PALMER, C.L.; CARLSON, J.R. y WITT, M. Data sharing, small science and institutional repositories. Philosophical Transactions of the Royal Society of London A. mathematical, Physical and Engineering Sciences, 2010, vol. 368, n 1926, p. 4023-4038. Disponible en: https://doi.org/10.1098/rsta.2010.0165.

DALTON, E.D.; TENOPIR, C. y BJÖEK, B-C. Attitudes of North American academics toward open access scholarly journals. Portal: Libraries and the academy, 2020, vol. 20, $\mathrm{n}^{\mathrm{o}}$ 1, p. 73-100. Disponible en: $<$ https://preprint.press.jhu.edu/portal/sites/ajm/files/20.1dalton.pdf $>$ [Consulta: 27 de enero de 2021]

DIGITAL SCIENCE. The state of open data 2020: the longest-running longitudinal survey and analysis on open data. London: Digital Science, 2020. Disponible en: <https:/www.digital-science.com/resources/portfolio-reports/thestate-of-open-data-2020/> [Consulta: 15 de enero de 2021]

FOSTER. The future of science is open. 2018. Disponible en: $<$ https://www.fosteropenscience.eu/ $>$ [Consulta: $23 \mathrm{de}$ enero de 2021]

FRITZ, L. y BINDER, C. Participation as relational space: A critical approach to analyzing participation in sustainability research. Sustainability, 2018, vol. 10, no 8, p. 2853. Disponible en: https://doi.org/10.3390/su10082853.

FU, D.Y. y HUGHEY, J.J. Releasing a preprint is associated with more attention and citations for the peer-reviewed article. eLife, 2019, vol. 8, e52646. Disponible en: <https://elifesciences.org/articles/52646> [Consulta: 28 de enero de 2021]

HERMAN, E. y NICHOLAS, D. Scholarly reputation building in the digital age: An activity-specific approach. Review article. El profesional de la información, 2019, vol. 28, $\mathrm{n}^{\mathrm{o}}$ 1, e280102. Disponible en: https://doi.org/10.3145/epi.2019.ene.02. 
JAMALI, H.R.; NICHOLAS, D. y HERMAN, E. Scholarly reputation in the digital age and the role of emerging platforms and mechanisms. Research Evaluation, 2015, vol. 24, p. 1-13. Disponible en: doi: 10.1093/reseval/rvv032. KIM, Y. Data sharing from the perspective of faculty in Korea. Libri, 2017, vol. 67, no 3, p. 179-192.

KIM, Y. y NAH, S. Internet researchers' data sharing behaviors: an integration of data reuse experience, attitudinal beliefs, social norms, and resource factors. Online information review, 2018, vol. 42, nº 1, p. 124-142.

LEVIN, N. y LEONELLI, S. How Does One "Open” science? Questions of Value in Biological Research. Science, Technology, \& Human Values, 2017, vol. 42, $\mathrm{n}^{\mathrm{o}}$ 2, p. 280-305. Disponible en: https://doi.org/10.1177\%2F0162243916672071.

LEVIN, N.; LEONELLI, S.; WECKOWSKA, D.; CASTLE, D. y DUPRÉ, J. How do scientists define openness? Exploring the relationship between open science policies and research practice. Bulletin of science, Technology \& Society, 2016, vol. 36, n 2, p. 128-141. Disponible en: https://doi.org/10.1177\%2F0270467616668760.

LUTHE, T. Success in transdisciplinary sustainability research. Sustainability, 2017, vol. 9, no 71 . Disponible en: https://doi.org/10.3390/su9010071.

MARCIAL, L.H. y HEMMINGER, B.M. Scientific data repositories on the Web: an initial survey. Journal of the American Society for information Science and Technology, 2010, vol. 61, $\mathrm{n}^{\mathrm{o}}$ 10, p. 2029-2048. Disponible en: https://doi.org/10.1002/asi.21339.

MCGUIRE, A.L.; HAMILTON, J.A.; LUNSTROTH, R.; MCCULLOUGH, L.B. y GOLDMAN, A. DNA data sharing. Research participants' perspectives. Genetics in Medicine, 2008, vol. 10, no 1, p. 46. Disponible en: $<$ https://www.nature.com/articles/gim20087> [Consulta: 23 de enero de 2021]

NICHOLAS, D. et al. Open science from the standpoint of the new wave of researchers: Views from the scholarly frontline. Information Services \& Use, 2019b, vol. 39, nº 4, p. 369 -374. Disponible en: https://doi.org/10.3233/ISU190069.

NICHOLAS, D. et al. New ways of building, showcasing, and measuring scholarly reputation. Learned Publishing, 2015, vol. 28, nº 3, p. 169-183. Disponible en: https://doi.org/10.1087/20150303.

NICHOLAS, D. et al. A global questionnaire survey of the scholarly communication attitudes and behaviours of early career researchers. Learned Publishing, 2020b, vol. 33, $\mathrm{n}^{\mathrm{o}}$ 3, p. 198-211. Disponible en: https://doi.org/10.1002/leap.

NICHOLAS, D. et al. How is open access publishing going down with early career researchers? An international, multidisciplinary study. El profesional de la información, 2020c, vol. 29, n ${ }^{\circ}$ 6, e290614. Disponible en: https://doi.org/10.3145/epi.2020.nov.14.

NICHOLAS, D. et al. Early career researchers and their publishing and authorship practices. Learned Publishing, 2017b, vol. 30, n 3, p. 205-2017. Disponible en: https://doi.org/10.1002/leap.1102.

NICHOLAS, D. et al. Does the scholarly communication system satisfy the beliefs and aspirations of new researchers? Summarizing the Harbingers research. Learned Publishing, 2020a, vol. 33, $\mathrm{n}^{\mathrm{o}}$ 2, p. 132-141. Disponible en: https://doi.org/10.1002/leap.1284.

NICHOLAS, D. et al. So, are early career researchers the harbingers of change? Learned Publishing, 2019a, vol. 32, $\mathrm{n}^{\circ}$ 3, p. 237-247. Disponible en: https://doi.org/10.1002/leap.1232.

NICHOLAS, D. et al. Early career researchers: Scholarly behaviour and the prospect of change. Learned Publishing, 2017a, vol. 30, nº 2, p. 157-166. Disponible en: https://doi.org/10.1002/leap.1098.

NOSEK, B.A. et al. Promoting an open research culture. Science, 2015, vol. 348, nº 6242, p. 1422-1425. Disponible en: https://doi.org/10.1126/science.aab2374.

O'HANLON, R.; MCSWEENEY, J. y STABLER, S. Publishing habits and perceptions of open access publishing and public access amongst clinical and research fellows. Journal of the Medical Library Association, 2020, vol. 108, $\mathrm{n}^{\mathrm{o}}$ 1, p. 47-58. Disponible en: https://doi.org/ 10.5195/jmla.2020.751.

PARK, J. y GABBARD, J.L. Factors that affect scientists' knowledge sharing behavior in health and life sciences research communities: differences between explicit and implicit knowledge. Computers in Human Behavior, 2018, vol. 78, p. 326-335. Disponible en: <https://dl.acm.org/doi/abs/10.1016/j.chb.2017.09.017> [Consulta: 4 de enero de 2021]

PIWOWAR, H.A. y CHAPMAN, W.W. Public sharing of research datasets: a pilot study of associations. Journal of informetrics, 2010, vol. $4, \quad \mathrm{n}^{\mathrm{o}} \quad 2, \quad$ p. $148-156 . \quad$ Disponible en: $<$ https://www.ncbi.nlm.nih.gov/pmc/articles/PMC3039489/> [Consulta: 28 de enero de 2021]

PIWOWAR, H. et al. The state of OA: a large-scale analysis of the prevalence and impact of Open Access articles. PeerJ, 2018, b6: e4375. Disponible en: https://doi.org/10.7717/peerj.4375.

ROBERTS, E.; ANDERSON, B.A.; SKERRATT, S. y FARRINGTON, J. A review of the rural-digital policy agenda from a community resilience perspective. Journal of Rural Studies, 2017, vol. 54, p. 372-385. Disponible en: https://doi.org/10.1016/j.jrurstud.2016.03.001.

RODRÍGUEZ-BRAVO, B. y NICHOLAS, D. Reputación y comunicación científica: investigadores españoles en el inicio de su carrera. El profesional de la información, 2019, vol. 28, $\mathrm{n}^{\mathrm{o}}$ 2, e280203. Disponible en: https://doi.org//10.3145/epi.2019.mar.03. 
RODRÍGUEZ-BRAVO, B. y NICHOLAS, D. Descubrir, leer, publicar, compartir y monitorizar el progreso: comportamiento de los investigadores junior españoles. El profesional de la información, 2020a, vol. 29, nº 5, e290503. Disponible en: https://doi.org/10.3145/epi.2020.sep.03.

RODRÍGUEZ-BRAVO, B. y NICHOLAS, D. Coautoría y revisión por pares: prácticas y percepciones de los investigadores noveles españoles. Revista General de Información y Documentación, 2020b, vol. 30, n 2 , p. 379399. Disponible en: http://dx.doi.org/10.5209/rgid.72817.

RUIZ-PÉREZ, S. y DELGADO-LÓPEZ-CÓZAR, E. Spanish researchers' opinions, attitudes and practices towards open access publishing. El profesional de la información, 2017, vol. 26, no 4, p. 722-734. Disponible en: https://doi.org/10.3145/epi.2017.jul.16.

SCHMIDT, L. y PRÖPPER, M. Transdisciplinarity as a real-world challenge: a case study on a north-south collaboration. Sustainability Science, 2017, vol. 12, p. 365-379. Disponible en: $<$ https://link.springer.com/article/10.1007/s11625-017-0430-8> [Consulta: 28 de enero de 2021]

SEGADO-BOJ, F.; MARTÍN-QUEVEDO, J. y PRIETO-GUTIÉRREZ, J.-J. Attitudes toward open Access, open peer review, and altmetrics among contributors to Spanish scholarly journals. Journal of scholarly publishing, 2018 , vol. 50, $\mathrm{n}^{\mathrm{o}}$ 1, p. 48-70. Disponible en: doi: 10.3138/jsp.50.1.08.

SEVERIN, A.; EGGER, M.; EVE, M.-P. y HÜRLIMANN, D. Discipline-specific open access publishing practices and barriers to change: an evidence-based review. F1000research, 2018, vol. 7, $\mathrm{n}^{\mathrm{o}} 1925$. Disponible en: $<$ https://f1000research.com/articles/7-1925> [Consulta: 26 de enero de 2021]

TAKASJIMA, K. et al. Ethical concerns on sharing genomic data including patients' family members. BMC medical ethics, 2018, vol. 19, $\mathrm{n}^{\circ}$ 61. Disponible en: <https://bmcmedethics.biomedcentral.com/articles/10.1186/s12910-0180310-5> [Consulta: 12 de enero de 2021]

TENOPIR, C. et al. Data sharing by scientists: practices and perceptions. PloS One, 2011, vol. 6, n 6, e21101. Disponible en: https://doi.org/10.1371/journal.pone.0021101.

TOELCH, U. y OSTWALD, D. Digital open-science-Teaching digital tools for reproducible and transparent research. PloS Biology, 2018, vol. 16, nº 7, e2006022. Disponible en: doi: 10.1371/journal.pbio.2006022.

VAN DEN EYNDEN, V. y CORTI, L. Advancing research data publishing practices for the social sciences: from archive activity to empowering researchers. International Journal on Digital libraries, 2017, vol. 18, $\mathrm{n}^{\circ} 2$, p. 113121. Disponible en: <https://link.springer.com/article/10.1007/s00799-016-0177-3> [Consulta: 11 de enero de 2021]

VICENTE-SAEZ, R. y MARTÍNEZ-FUENTES, C. Open Science now: A systematic literature review for an integrated definition. Journal of Business Research, 2018, vol. 88, p. 428-436. Disponible en: https://doi.org/10.1016/j.jbusres.2017.12.043.

WEBER, N.M.; PIWOWAR, H.A. y VISION, T.J. Evaluating data citation and sharing policies in the environmental sciences. Proceedings of the American Society for information Science and Technology, 2010, vol. 47, $\mathrm{n}^{\circ}$ 1, p. 1-2. Disponible en: https://doi.org/10.1002/meet.14504701445.

WOUTERS, P. Policies on digital research data: an international survey. Amsterdam: Niwi-Knaw, 2002.

$\mathrm{XU}$, J. et al. Chinese researchers' perceptions and use of open access journals. Results of an online questionnaire survey. Learned publishing, 2020, vol. 33, n 3, p. 246-258. Disponible en: https://doi.org/10.1002/leap.1291.

ZENK-MÖLTGEN, W.G; AKDENIZ, E.; KATSANIDOU, A.; NABHOVEN, V. y BALABAN, E. Factors influencing the data sharing behavior of researchers in sociology and political science. Journal of Documentation, 2018, vol. 74, $\mathrm{n}^{\circ}$ 5, p. 1053-1073. Disponible en: doi:10.1108/JD-09-2017-0126. 
¿Publicas artículos en acceso abierto?

- Sí, frecuentemente.

- Sí, ocasionalmente.

- No.

Según tu experiencia, ¿hasta qué punto las siguientes afirmaciones constituyen una ventaja de publicar artículos en acceso abierto?

- Incrementa la visibilidad/el descubrimiento.

- La audiencia potencial es más amplia y más numerosa.

- Mayores posibilidades de conectividad/networking.

- Mejora la colaboración-la oferta de oportunidades.

- Incrementa el impacto en términos de descargas, lecturas, citas, menciones en redes sociales.

- Las revistas en acceso abierto publican más rápido, acortan los plazos de respuesta.

- Cumplimiento con los mandatos de la universidad o de los financiadores.

- Contribuyen a que los avances científicos sean más rápidos.

Según tu experiencia, ¿hasta qué punto las siguientes afirmaciones constituyen una desventaja de publicar artículos en acceso abierto?

- La percepción de que las revistas en acceso abierto tienen una calidad pobre.

- La percepción de que las revistas en acceso abierto tienen un prestigio/estatus inferior.

- $\quad$ El coste de publicar en acceso abierto.

- Los riesgos que supone para el progreso de la carrera investigadora y para la reputación.

- La posibilidad de que las revistas en acceso abierto puedan ser plagiadas más fácilmente.

- La existencia de demasiadas revistas depredadoras.

¿Has producido datos?

- Sí.

- No.

Si tu respuesta es afirmativa ¿los has dejado disponibles en abierto?

- Sí.

- No.

¿Cómo los has dejado accesibles en abierto?

- Publicando los datos como materiales suplementarios de un artículo.

- Publicando un data paper con el conjunto de datos.

- Alojando los datos en un sitio web. Con archivos disponibles para ser descargados.

- Alojando los datos en un repositorio como Dryad, Figshare y Zenodo.

¿Por qué decidiste dejarlos accesibles en abierto?

- Permite la reproducibilidad.

- Permite la reutilización.

- Asegura su preservación y su futura accesibilidad.

- Estimulado/obligado por las políticas de la ciencia abierta.

- Para cumplir con la política de publicación de la revista.

- Confiere ventajas en la obtención de citas.

- Es una señal de credibilidad.

- Facilita la colaboración.

- Compromiso con las políticas del acceso abierto.

- Otras razones.

Si tu respuesta es negativa, ¿por qué no los dejas accesibles en abierto?

- Preocupación por la competencia. 
- Riesgo para el desarrollo de mi carrera dado que compartir datos no es recompensado por los sistemas de evaluación actuales.

- $\quad$ No hay mandatos que obliguen a compartir datos.

- Supone demasiado trabajo limpiar los datos.

- $\quad$ El tamaño de los archivos de datos impide compartirlos.

- La naturaleza de los datos (por ejemplo, su carácter confidencial o relacionado con la seguridad) impide compartirlos.

- Otras razones. 